UG-FT-302/13

CAFPE-172/13

\title{
Contamination of Dark Matter Experiments from Atmospheric Magnetic Dipoles
}

\author{
A. Bueno, M. Masip, P. Sánchez-Lucas, N. Setzer \\ CAFPE and Departamento de Física Teórica y del Cosmos \\ Universidad de Granada, 18071 Granada, Spain \\ abueno, masip, patriciasl, nsetzer@ugr.es
}

\begin{abstract}
Dark matter collisions with heavy nuclei (Xe, Ge, Si, Na) may produce recoils observable at direct-search experiments. Given that some of these experiments are yielding conflicting information, however, it is worth asking if physics other than dark matter may produce similar nuclear recoils. We examine under what conditions an atmospherically-produced neutral particle with a relatively large magnetic dipole moment could fake a dark matter signal. We argue that a very definite flux could explain the signals seen at DAMA/LIBRA, CDMS/Si and CoGeNT consistently with the bounds from XENON100 and CDMS/Ge. To explore the plausibility of this scenario, we discuss a concrete model with $10-50 \mathrm{MeV}$ sterile neutrinos that was recently proposed to explain the LSND and MiniBooNE anomalies.
\end{abstract}




\section{Introduction}

The current tune of dark matter (DM) experiments is that of discordance: DAMA/LIBRA [1, 2], CoGeNT [3], CRESST-II [4], and CDMS/Si [5] report signals while XENON100 and the rest of experiments see nothing. In addition, the signal seen by DAMA/LIBRA is seemingly inconsistent with the ones at CoGeNT and CDMS (at least when assuming a WIMP as DM). Given this cacophonous state, it seems reasonable to consider alternative explanations for the current experimental data. In particular, the fact that the DAMA/LIBRA modulation closely follows ${ }^{1}$ that of the muon flux [8] suggests that it may be atmospheric in origin. This idea is not new, as a number of authors have proposed that the two phenomena are related either directly [9, 10] or indirectly through neutron backgrounds [8. In this work we take an orthogonal approach and consider the possibility that these observations may be a genuine sign of new physics, just not DM. Thus, we address the question of whether direct search experiments exclusively probe DM or whether they could be revealing a contamination by some unexpected physics.

To obtain a likely new physics candidate, one starting point is to consider standard background sources and modify them in order to increase or tune their effects. The usual set-up for a direct-search experiment is to have some target mass and look for recoils off a nucleus. Therefore, in these experiments, ambient neutrinos that interact coherently with the nucleus via $Z$-exchange represent one such irreducible background. In Figure 1(right) we plot our estimate [1] for this neutrino background at XENON100 [12] and CDMS/Si [5] (whose lighter target implies larger recoils $T$ ) for the neutrino flux given in Figure 11(left). At energies below $20 \mathrm{MeV}$ the ambient flux is dominated by solar neutrinos whereas at higher energies neutrinos come from the decay of atmospheric muons and mesons. At $E \approx 2$ $\mathrm{MeV}$ there is a significant contribution to the flux from geoneutrinos produced by radiactive ${ }^{238} \mathrm{U},{ }^{235} \mathrm{Th}$ and ${ }^{40} \mathrm{~K}[13$, but this contribution is not important in direct-search experiments (Figure 1, right). Solar neutrinos produce $\mathcal{O}(100)$ events per year and ton of ${ }^{131} \mathrm{Xe}$, but all of them are below threshold for XENON100. In CDMS/Si solar neutrinos yield $\mathcal{O}(100)$ events per year and ton, but the experimental threshold only admits $\mathcal{O}(10)$ of these. The contribution of atmospheric neutrinos at $10^{-3} / \mathrm{yr} /$ ton in XENON100 and $10^{-4} / \mathrm{yr} /$ ton at CDMS is far too low to be significant; however, this need not be the case if there are other neutrino species that are produced frequently in muon decays.

\footnotetext{
${ }^{1}$ Assuming a simple $A \cos \left[\omega\left(t-t_{0}\right)\right]$ dependence, one obtains $t_{0}=185 \pm 15$ days for the muon flux [6] and $t_{0}=146 \pm 7$ days in DAMA/LIBRA [2] indicating an incompatibility of a common phase by 2 standard deviations. A further investigation of the correlation is given in [7. We think that a dedicated study of the correlation between atmospheric parameters (such as temperature and pressure) and the DAMA/LIBRA signal could provide evidence of an atmospheric origin if this is indeed the case.
} 

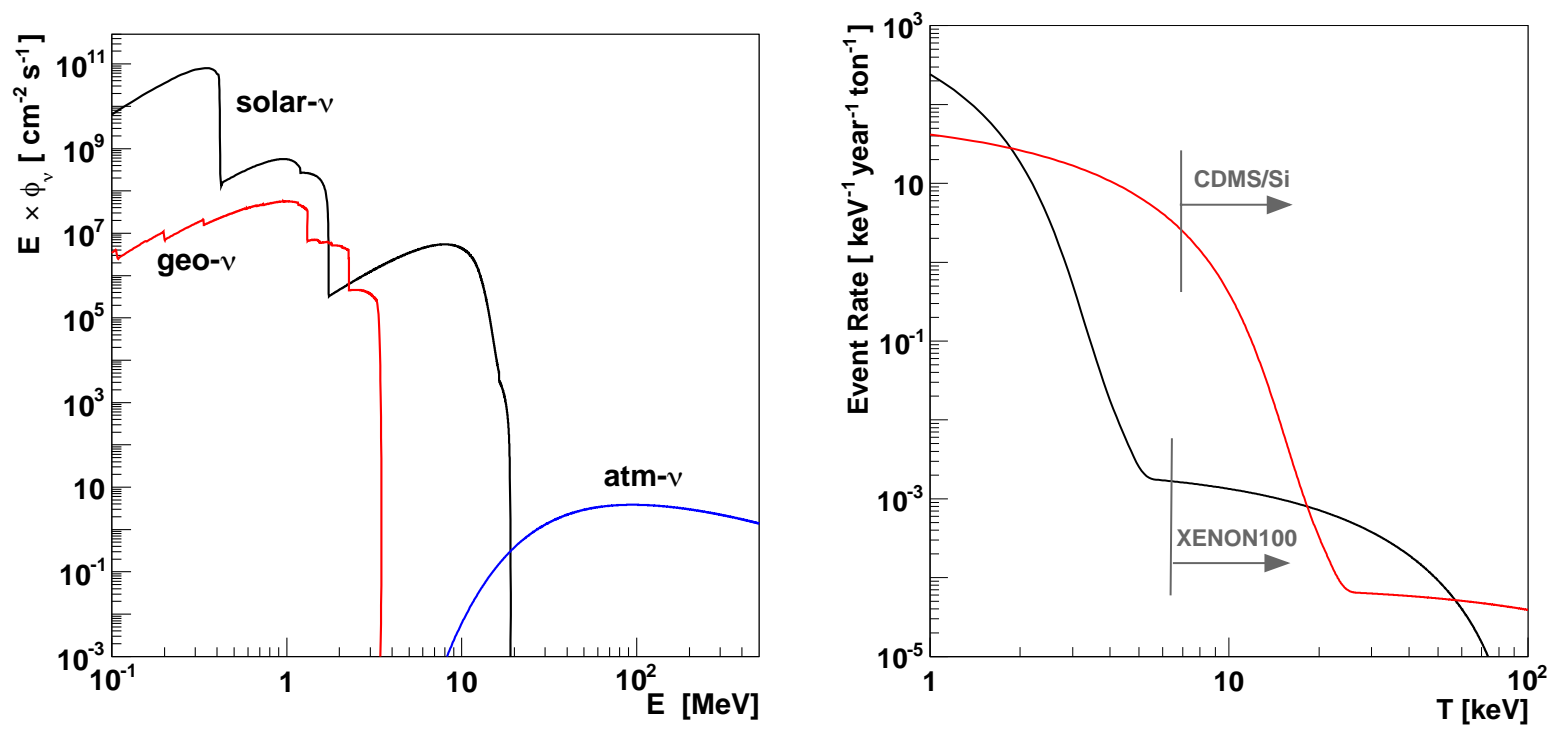

Figure 1: To the left is shown the ambient neutrino fluxes; to the right the corresponding recoil distribution that they produce at XENON100 [12] and CDMS/Si [5], with the energy threshold of each experiment also indicated. The recoil distribution has a "kink" which represents the transition from solar neutrinos to atmospheric neutrinos (the geoneutrino contribution is not significant). For the complete details on obtaining these plots see [11].

The above then suggests a way to introduce new physics: a sterile neutrino or, generically, a new neutral particle produced either atmospherically or in the sun. Sterile neutrinos of solar origin have been explored previously [14, 15], so we will focus on atmospheric production. We provide the details and properties of such a hypothetical, atmospherically-produced particle in Section 2. In Section 3, we discuss how this particle appears at various DM experiments. In Section 4 we introduce a model that serves as a concrete example of this particle. Finally, we conclude our discussion in Section 5.

\section{Magnets from the Atmosphere}

Starting from the idea of an additional neutrino species, we contemplate the properties that a hypothetical particle should have to generate a signal at DM experiments. A likely candidate would possess the following properties:

- neutral charge 
- produced in muon decays (directly or within a decay chain) with frequency of 1 particle per 100-1000 decays

- mass in excess of $10 \mathrm{MeV}$

- lifetime between $10^{-6} \mathrm{~s}$ and $1 \mathrm{~s}$

- large magnetic dipole moment

The requirement of neutral charge is evident for mimicking a DM signal. The second property links the atmospheric production to muons, so that the hypothetical particle's flux may modulate just as the muon flux - thus providing the possibility to explain annual modulations. The condition on its mass permits the particle to avoid astrophysical constraints (for example production in the sun or other stars), while the lifetime range affords the possibility of being atmospherically produced and still reaching the detector without introducing cosmological problems (such as disrupting big-bang nucleosynthesis). The final condition of a large magnetic dipole moment provides a means of giving the particle a long-range electromagnetic (EM) interaction without introducing another gauge symmetry.

An EM dipole will introduce photon-mediated interactions with nuclei that are rather different from the standard neutrino interactions and thus allow for a significant contamination in DM experiments. Elastic neutrino interactions are mediated by a $Z$ boson and as such are short-distance processes. If we ignore the nuclear form factor, such interactions tend to give nuclear recoils, $T$, right below

$$
T_{\max }=\frac{2 M\left(E^{2}-m^{2}\right)}{M^{2}+2 M E+m^{2}},
$$

with $E$ the energy of the neutrino, $m$ its mass, and $M$ the mass of the nucleus. Furthermore, the form factor will suppress elastic collisions with recoils having a momentum transfer $q^{2}=2 M T \gtrsim(100 \mathrm{MeV})^{2} ;$ therefore,

$$
T_{\max }^{\prime}=\frac{(0.1 \mathrm{GeV})^{2}}{2 M} .
$$

These $Z$-mediated processes will then be dominated by recoils near $\min \left\{T_{\max }, T_{\max }^{\prime}\right\}$, with lower recoils giving a non-significant contribution to the total cross section. In contrast, the photon-mediated interaction of a dipole includes all recoils below $\min \left\{T_{\max }, T_{\max }^{\prime}\right\}$ : all of them contribute equally $\left.\right|^{2}$ to the total cross section.

\footnotetext{
${ }^{2}$ The atomic form factor will suppress the cross section also at very low recoils.
} 
We therefore introduce our hypothetical particle as a Dirac fermion, $\Psi$, with a magnetic dipole moment $\mu$. Its lagrangian is then

$$
\mathcal{L} \supset \frac{1}{2} \mu \bar{\Psi} \sigma_{\alpha \beta} \Psi F^{\alpha \beta}+m \bar{\Psi} \Psi
$$

where $F^{\mu \nu}$ is the field strength for the photon. With the langragian of Eq. (3), we may now discuss how this particle, a sterile Dirac neutrino, could be seen at direct-search DM experiments.

\section{Contaminating Dark Matter Experiments}

With the lagrangian given in Eq. (3), our particle will collide with nuclei through an EM dipole interaction: assuming a spin-1/2 target of mass $M$ and charge $Z$, the differential cross-section for the elastic scattering reads

$$
T \frac{d \sigma}{d T}=\alpha Z^{2} \mu^{2} F_{Z}^{2}\left(q^{2}\right)\left(1-\frac{T}{2 M} \frac{2 E M-m^{2}}{E^{2}-m^{2}}\right),
$$

where $\alpha$ is the fine-structure constant and we take the form factor [16]

$$
F_{Z}^{2}\left(q^{2}\right)= \begin{cases}\frac{a^{4} q^{4}}{\left(1+a^{2} q^{2}\right)^{2}} & q^{2} \leq t_{0}=7.39 m_{e}^{2} \\ \frac{1}{\left(1+q^{2} / d\right)^{2}} & q^{2}>t_{0}\end{cases}
$$

with $a=111.7 \mathrm{MeV}^{-1} Z^{-1 / 3} / m_{e}$ and $d=0.164 A^{-2 / 3} \mathrm{GeV}^{2}$. As we mentioned, the form factor suppresses elastic collisions of $q^{2}=2 M T \gtrsim(100 \mathrm{MeV})^{2}$ since at these energies inelastic processes dominate.

The differential cross section is plotted in Figure 2 for four target elements and several incident energies. We have assumed that the particle has a mass of $m=10 \mathrm{MeV}$ and a magnetic dipole moment $\mu=10^{-6} \mu_{B}$ (we show later that this magnetic moment is the largest obtainable in the natural embedding of this setup). As can be seen in Figure 2, at large energies $(E \sim 100 \mathrm{MeV})$ the maximum recoil is determined solely by the nuclear form factor; at low energies $(E \sim \mathcal{O}(10) \mathrm{MeV})$, however, kinematics force the differential cross section to zero before the form factor becomes relevant. It is this kinematic feature that can be used to explain the various experiments.

Given that $T \frac{d \sigma}{d T} \approx$ const, an incident particle of energy $E$ will interact at a given detector with a visible cross section (i.e., $\left.T \geq T_{\text {thres }}\right)$ of

$$
\sigma(E) \approx \alpha Z^{2} \mu^{2} \ln \frac{T_{\max }(E)}{T_{\text {thres }}} .
$$



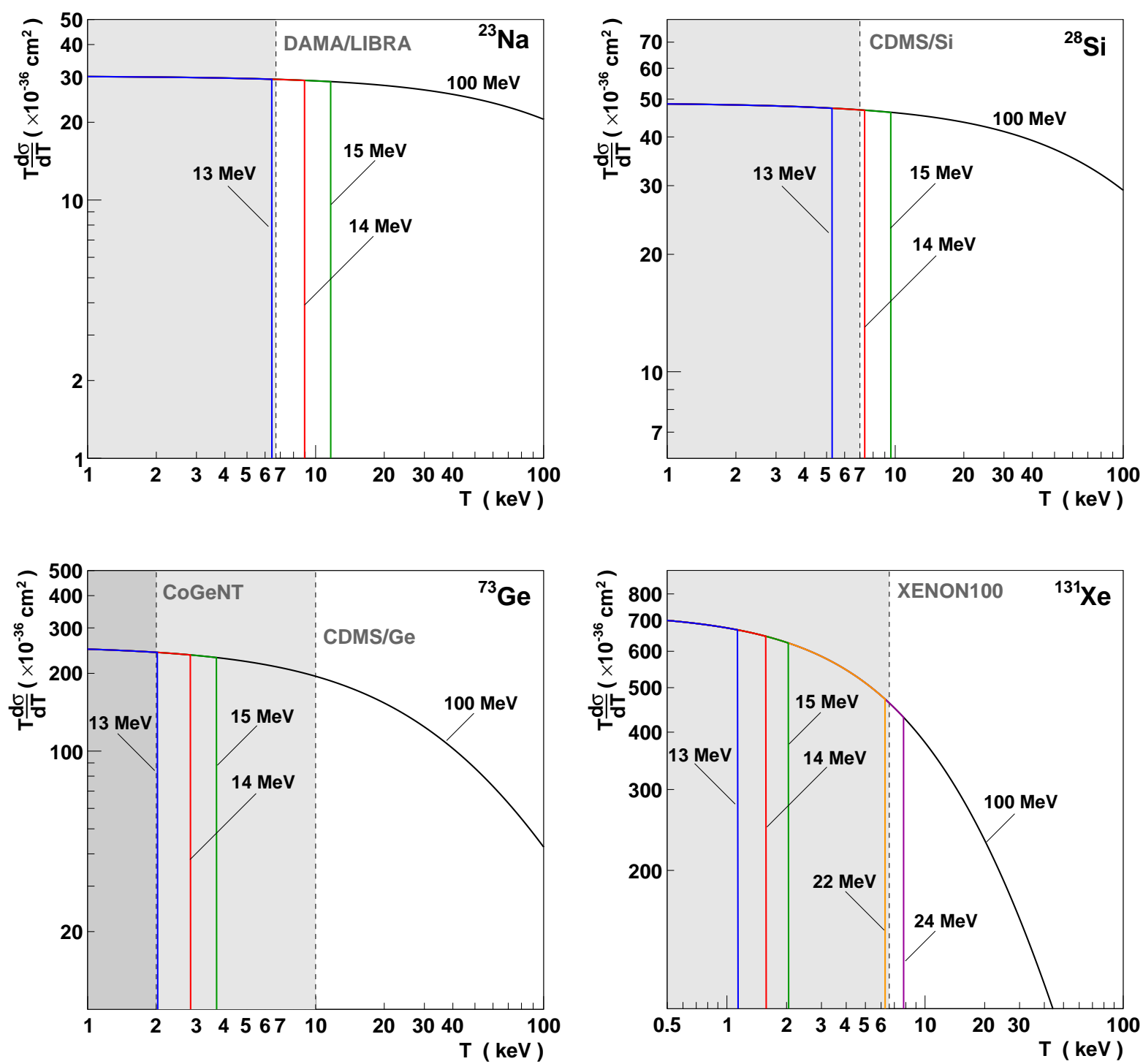

Figure 2: The recoil times the differential cross section versus the recoil of an elastic collision of our hypothetical particle with four different target nuclei. The shaded regions denote recoils below threshold for the indicated experiments. The mass of the particle is taken to be $m=10 \mathrm{MeV}$ and its magnetic moment is $\mu=10^{-6} \mu_{B}$. Note that a particle with an energy between 13 and $14 \mathrm{MeV}$ can be seen by DAMA/LIBRA and CoGeNT, but not CDMS or XENON100. For an energy between 14 and $15 \mathrm{MeV}$ the particle can be seen by CDMS/Si, but not XENON100; it is not until the energy of the particle is between 22 and $24 \mathrm{MeV}$ that it can be seen by XENON100. 


\begin{tabular}{|c|c|c|c|c|c|}
\hline Experiment & Threshold $(\mathrm{keV})$ & Exposure $(\mathrm{kg} \mathrm{d})$ & Material & Tgt. & Num. Events \\
\hline DAMA/NaI[2] & 6.7 & 109500 & $\mathrm{NaI}(\mathrm{Tl})$ & $\mathrm{Na}$ & $\sim 4300$ \\
DAMA/LIBRA[2] & 6.7 & 317697 & $\mathrm{NaI}(\mathrm{Tl})$ & $\mathrm{Na}$ & $\sim 12400$ \\
CoGeNT[3] & 2 & 18.48 & $\mathrm{Ge}$ & $\mathrm{Ge}$ & $\sim 7-13$ \\
CDMS/Si[5] & 7 & 140.2 & $\mathrm{Si}$ & $\mathrm{Si}$ & 3 \\
CDMS/Ge[17] & 10 & 612 & $\mathrm{Ge}$ & $\mathrm{Ge}$ & 0 \\
XENON100[12] & 6.6 & 7636.4 & $\mathrm{Xe}$ & $\mathrm{Xe}$ & 0 \\
\hline
\end{tabular}

Table 1: The experimental data (taken from papers indicated) and the target nuclei of interest. Note that CoGeNT does not directly give the number of events; however, in Fig. 3 of [3] they plot the allowed number of events for a dark matter WIMP of mass 7 and 10 $\mathrm{MeV}$. It is from these curves that we estimate the allowed number of events shown above.

As a consequence, for a given detector exposure, $X$, a flux of $N(E)=\Phi(E) \Delta E$ of such particles reaching the detector will produce

$$
N_{\text {events }} \approx \alpha Z^{2} \mu^{2} \frac{X}{m_{\text {mat }}} N(E) \ln \frac{T_{\max }(E)}{T_{\text {thres }}}
$$

where $\Phi(E)=d N / d A d t d E$ is the differential flux (per unit area, time, and energy) and $m_{\text {mat }}$ is the mass of the target material in the detector (either the atomic or molecular mass in $\mathrm{kg}$ to match the units of the exposure). If we bin our flux in $1 \mathrm{MeV}$ increments, the plots of Figure 2 show that the energies between 13-14 MeV introduce recoils that are seen both at DAMA/LIBRA and CoGeNT. Taking the targets and exposures in Table 1 (and an average energy in the bin of $E=13.5 \mathrm{MeV}$ ), we predict the ratio

$$
\frac{N_{\text {events }}^{\text {DAMA/LIBRA }}}{N_{\text {events }}^{\text {CoGeNT }}} \sim 700 \sim \frac{12400}{17}
$$

for a mass $m=10 \mathrm{MeV}$ and independent of the magnetic dipole $\mu$. This should be compared to the values from the actual experiments, which are also given in Table 1. Note that the prediction of Eq. (8) is fairly well-obeyed by the data.

While the 13-14 MeV bin is seen by both DAMA/LIBRA and CoGeNT, the experiments CDMS and XENON100 only see higher-energy particles due to their heavier targets or higher thresholds. For CDMS/Si, the $14-15 \mathrm{MeV}$ bin represents the lowest neutrino energies yielding detectable recoils. If we take the number of DAMA/LIBRA events as the base of comparison, the ratio of events at CDMS/Si to DAMA/LIBRA is given by $N(14.5 \mathrm{MeV}) / N(13.5 \mathrm{MeV})$ - a quantity fixed due to the 3 events at CDMS/Si. With the 
assistance of Eq. (7), this events ratio sets the ratio of the fluxes,

$$
\frac{\Phi(14.5 \mathrm{MeV})}{\Phi(13.5 \mathrm{MeV})} \sim 0.045
$$

independently of $\mu$. Similarly, the $22-24 \mathrm{MeV}$ bin is the first one producing nuclear recoils above the threshold at XENON100. As XENON100 does not see any events, we obtain an upper bound on the ratio of fluxes,

$$
\frac{\Phi(23.5 \mathrm{MeV})}{\Phi(14.5 \mathrm{MeV})} \lesssim 2.9 \times 10^{-4}
$$

which implies that XENON100 would see 0.1 event (i.e., none) for every 3 observed by CDMS/Si. It should be emphasized that, given Eqs. (9) and (10), neither DAMA/LIBRA nor CoGeNT would see any significant signal coming from dipoles with energies in excess of $14 \mathrm{MeV}$. In fact, all experiments would only see a significant number of events near their energy thresholds.

It is possible to further constrain the flux if an exponential dependence on momentum ${ }^{3}$ is assumed,

$$
\Phi \propto\left(E^{2}-m^{2}\right)^{-\gamma} .
$$

With this ansatz, the ratio of Eq. (9) implies the rather steep fall-off of $\gamma \approx 10$. Taking the flux to obey this in the entire region of interest implies that

$$
\frac{N_{\text {events }}^{\mathrm{XENON} 100}}{N_{\text {events }}^{\mathrm{CDMS}}} \sim 7.4 \times 10^{-5}
$$

so that the ratio of events at XENON100 to CDMS/Si is much less than the bound of Eq. (10) - actually, XENON100 would need about 1000 years of running time to get just one event. While this rapid fall-off is consistent with current experiments, it seems difficult to reconcile with an atmospheric production in muon decays. Since the details of how the spectrum may be shifted to lower energies are rather model-specific, we defer that discussion until the next section. In any case, it is worth pointing out that the functional dependence of the flux is entirely unknown and would not be determined by the dark matter experiments.

\section{A Concrete Model: The Heavy Neutrino}

Having established the generic properties that our dipole should have in order to consistently explain direct-search DM experiments, it is time to introduce an example model. As previously mentioned, massive sterile (gauge singlet) neutrinos are a natural possibility: they can

\footnotetext{
${ }^{3}$ Typically the literature refers to a flux dependent on energy rather than momentum; however, this assumes that $E \gg m$ so that $|\vec{p}| \approx E$. Here we have $E \sim m$ so it is necessary to distinguish the two.
} 
mix with the muon neutrino flavor and appear in a fraction of muon decays. In fact, although the three-flavor picture fits the solar, atmospheric, and reactor data [18] well, there has been a persistent anomaly in experiments with neutrino beams from particle accelerators. Both LSND [19 22] and MiniBooNE [23 26] have observed an excess of 3 events with an electron in the final state per each 1000 charged current (CC) $\nu_{\mu}$ interactions. These excesses may be explained by the addition of two sterile neutrinos with masses around $1 \mathrm{eV}$ [27] or in the 10 's of MeV [28, 29]. The model we introduce shall be based upon this latter interpretation, which is also able to interprete the negative result at KARMEN [30].

Our basic set up will be defined by two Dirac neutrinos, $\nu_{h}$ and $\nu_{h^{\prime}}$, in addition to the three standard flavors (which we assume to be Majorana particles). We will use hereafter a two-component spinor notation, with $f$ and $f^{c}$ indicating fermion and antifermion fields both of left-handed chirality. We have then

$$
\nu_{h}=\left(\begin{array}{c}
N_{h} \\
\left(N_{h}^{c}\right)^{\dagger}
\end{array}\right) \quad \nu_{h^{\prime}}=\left(\begin{array}{c}
N_{h^{\prime}} \\
\left(N_{h^{\prime}}^{c}\right)^{\dagger}
\end{array}\right) .
$$

We will assume that $N_{h}$ (the left-handed component of $\nu_{h}$ ) mixes with the muon-neutrino, $\nu_{\mu}$; the effective lagrangian should thus contain

$$
-\mathcal{L}_{\text {eff }} \supset \frac{y v_{\mathrm{wk}}}{\sqrt{2}} \nu_{\mu} N_{h}^{c}+m_{h} N_{h} N_{h}^{c}+m_{\nu_{\mu}} \nu_{\mu} \nu_{\mu}+\text { h.c. }
$$

The mass eigenstates are then given by

$$
N_{h}^{\prime}=N_{h} \cos \theta+\nu_{\mu} \sin \theta, \quad \nu_{\mu}^{\prime}=-N_{h} \sin \theta+\nu_{\mu} \cos \theta,
$$

where (neglecting corrections of $\left.\mathcal{O}\left(m_{\nu_{\mu}} / m_{h}\right)\right)$

$$
\sin \theta=\frac{y v_{\mathrm{wk}}}{\sqrt{2 m_{h}^{2}+y^{2} v_{\mathrm{wk}}^{2} / 2}} \approx U_{\mu h}
$$

In order to reproduce the LSND anomaly [28] the model requires $m_{h} \approx 50 \mathrm{MeV}$ and $\left|U_{\mu h}\right|^{2}=0.003$ (which is obtained for $y v_{\mathrm{wk}} / \sqrt{2} \approx 2.7 \mathrm{MeV}$ ). In addition, explaining the MiniBooNE anomaly consistently with T2K data [31] requires the neutrino $\nu_{h}$ to decay with $99 \%$ branching ratio into another sterile neutrino [29] - namely $\nu_{h^{\prime}}$-through the process

$$
\nu_{h} \rightarrow \nu_{h^{\prime}} \gamma
$$

This decay is induced by the operator

$$
\mathcal{L}_{\text {eff }} \supset \frac{1}{2} \mu_{\mathrm{tr}}^{h h^{\prime}} N_{h}^{c} \sigma_{\alpha \beta} N_{h^{\prime}} F^{\alpha \beta}+\text { h.c. },
$$


which yields the rather short lifetime $\tau_{h} \approx 5 \times 10^{-9} \mathrm{~s}$ for $\mu_{\mathrm{tr}}^{h h^{\prime}} \approx 2.4 \times 10^{-8} \mu_{B}$. Thus, as $\nu_{h}$ would be produced in $0.1-1 \%$ of all muon and kaon decays [32], $\nu_{h^{\prime}}$ would be very abundant in the atmosphere provided its lifetime is sufficiently long.

The above supplies a plausible production mechanism for the $\nu_{h^{\prime}}$, but we still have to justify the relatively large value of the EM transitions and the magnetic moments. We will first show that the required transitions, $\mu_{\mathrm{tr}}^{i h}$, can be naturally obtained in left-right [33] extensions of the standard model (which provide a natural embedding for these sterile neutrinos), but that the simplest extension does not imply a large enough magnetic moment $\mu_{h^{\prime}}$. We will then discuss a possible scenario that could boost the value of this magnetic moment.

Let us assume a left-right model where the sterile neutrinos, $\left\{N_{h}, N_{h}^{c}\right\}$ and $\left\{N_{h^{\prime}}, N_{h^{\prime}}^{c}\right\}$ are accommodated in $S U(2)_{R}$ doublets together with charged leptons,

$$
L_{h}=\left(\begin{array}{c}
N_{h} \\
E_{h}^{-}
\end{array}\right) \quad L_{h}^{c}=\left(\begin{array}{c}
E_{h}^{c+} \\
N_{h}^{c}
\end{array}\right) \quad L_{h^{\prime}}=\left(\begin{array}{c}
N_{h^{\prime}} \\
E_{h^{\prime}}^{-}
\end{array}\right) \quad L_{h^{\prime}}^{c}=\left(\begin{array}{c}
E_{h^{\prime}}^{c+} \\
N_{h^{\prime}}^{c}
\end{array}\right) .
$$

It is straightforward to define a framework where the breaking of $S U(2)_{R}$ gives large masses of order $v_{R}$ to the charged components of these doublets while the sterile neutrinos remain light; that is, it is possible to get $m_{h}=50 \mathrm{MeV}, m_{h^{\prime}}=10 \mathrm{MeV}$, while $m_{E} \approx 500 \mathrm{GeV}$. The mass matrix of the charged leptons, $m_{i j}^{E} E_{i} E_{i}^{c}\left(i, j \in\left\{h, h^{\prime}\right\}\right)$, is then what gives rise to the decay of $\nu_{h}$ along with the magnetic dipole moment of $\nu_{h^{\prime}}$ : these EM transitions will be generated by the processes shown in Figure 3. In particular, the $\nu_{h} \rightarrow \nu_{h^{\prime}} \gamma$ decay is induced by $i=h, j=h^{\prime}$ while the magnetic dipole moment is gotten for $i=j=h^{\prime}$. To reproduce the scenario discussed in [29], it is necessary that $m_{i j}^{E}$ obey the hierarchy $m_{h h^{\prime}}^{E} \ll m_{h^{\prime} h}^{E} \approx 0.5 m_{h h}^{E} \ll m_{h^{\prime} h^{\prime}}^{E}$. For the magnetic dipole moment $\mu_{h^{\prime}}$ of $\nu_{h^{\prime}}$ we obtain

$$
\mu_{h^{\prime}}=\frac{e g_{R}}{4 \pi^{2} v_{R}} r \frac{r^{4}+12 r^{2}-13+\left(6 r^{4}-20 r^{2}\right) \log r^{2}}{8\left(r^{2}-1\right)^{3}},
$$

with $r=m_{E} / M_{R}$ and taking the limit $m_{h^{\prime}} \ll m_{E}, M_{R}$. Values around $r=1.5, v_{R}=$ $500 \mathrm{GeV}$, and $g_{R}=0.8$ can yield $\mu_{h^{\prime}} \approx 10^{-5} \mathrm{GeV}^{-1}=0.3 \times 10^{-6} \mu_{B}$. While this magnetic moment is relatively large for a neutral particle, it is not large enough to explain the number of events at DAMA/LIBRA without a significant increase in the flux (recall that Eq. (7) states that the number of events is proportional to $\left.\mu^{2} \Phi\right)$. Unfortunately, this magnetic moment seems close to the maximum value that a loop of exotic charged particles can generate without appealing to either strong dynamics or higher multiplicities. As such, another means of generating large magnetic moments will be required if the flux is to remain a fraction of that of $\nu_{\mu}$. 

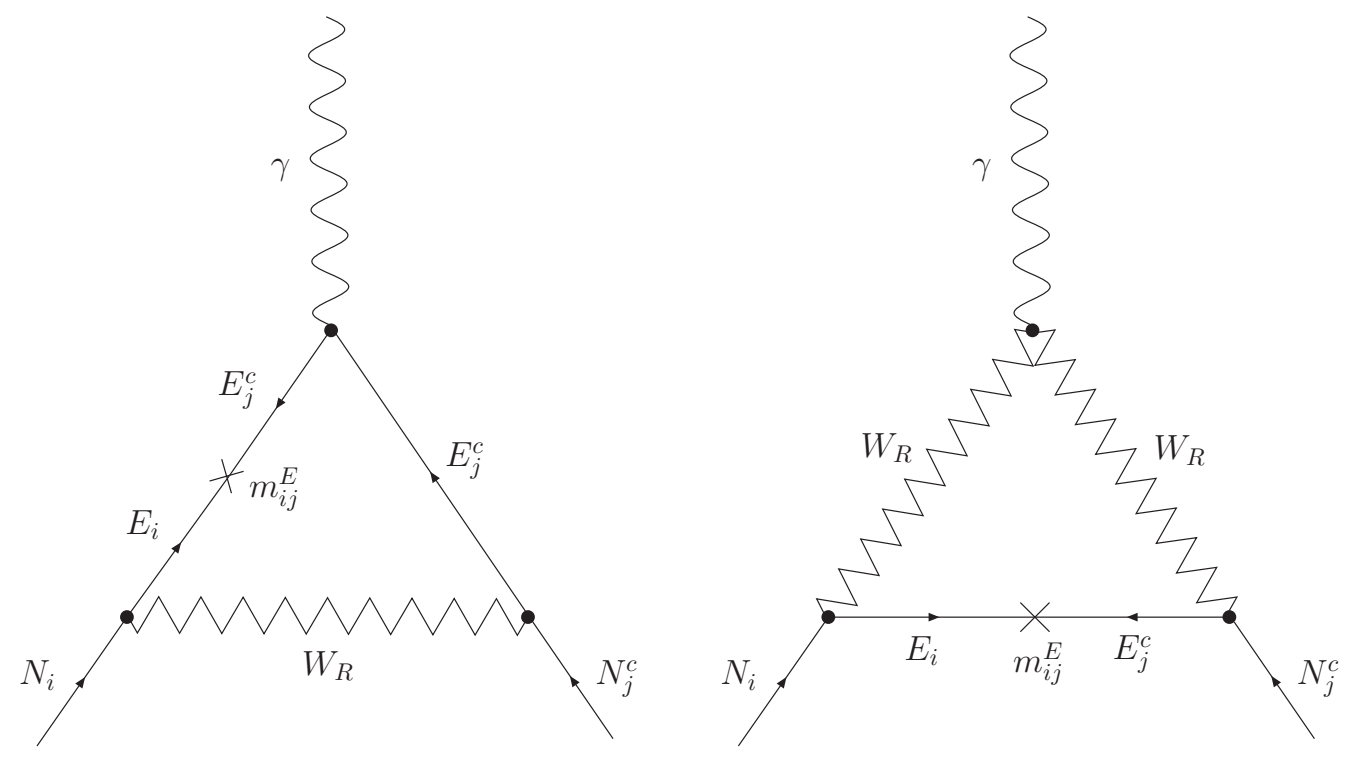

Figure 3: The one-loop diagram generating an electromagnetic transition between $N_{i}$ and $N_{j}^{c}$ in the left-right model discussed in the text.

Before considering the enhancement of the dipole moment, it is useful to estimate the impact of this setup on DM experiments. We will assume that the total number of atmospheric heavy neutrinos, $\nu_{h^{\prime}}$, is $0.5 \%$ that of muon neutrinos, and will take the muon neutrino abundance (in Figure 1) from [34]. For the energy distribution we will take

$$
\Phi_{h^{\prime}}(E) \propto \begin{cases}E & m_{h^{\prime}} \leq E<40 \mathrm{MeV} \\ E^{-2.7} & E \geq 40 \mathrm{MeV}\end{cases}
$$

We will set $m_{h^{\prime}}=10 \mathrm{MeV}$ and consider a lifetime long enough so that we can ignore $\nu_{h^{\prime}}$ decays.

With the above criteria, the expected number of $\nu_{h^{\prime}}$ events per year and ton at XENON100 and CDMS/Si is shown in Figure 4 for $\mu_{h^{\prime}}=10^{-6} \mu_{B}$ (dotted) and $\mu_{h^{\prime}}=10^{-5} \mu_{B}$ (solid). For comparison, we also include the number of events from standard neutrinos along with the current XENON100 limit[12] of a $55 \mathrm{GeV}$ WIMP having a cross section of $\sigma_{\chi N}=2 \times 10^{-45} \mathrm{~cm}^{2}$. The figure demonstrates that although the number of $\nu_{h^{\prime}}$ events that we obtain is larger than the background from ambient neutrinos, an observable signal at both XENON100 and CDMS/Si would require magnetic moments around $10^{-5} \mu_{B}$ rather than the $10^{-6} \mu_{B}$ generated in the left-right symmetric model (specifically, for $\mu_{h^{\prime}}=10^{-5} \mu_{B}$ we estimate 171 events per year and ton at XENON100 and 208 at CDMS/Si). Even if one could obtain a magnetic dipole moment of $10^{-5} \mu_{B}$, it would still be too small to have an impact on DAMA/LIBRA 

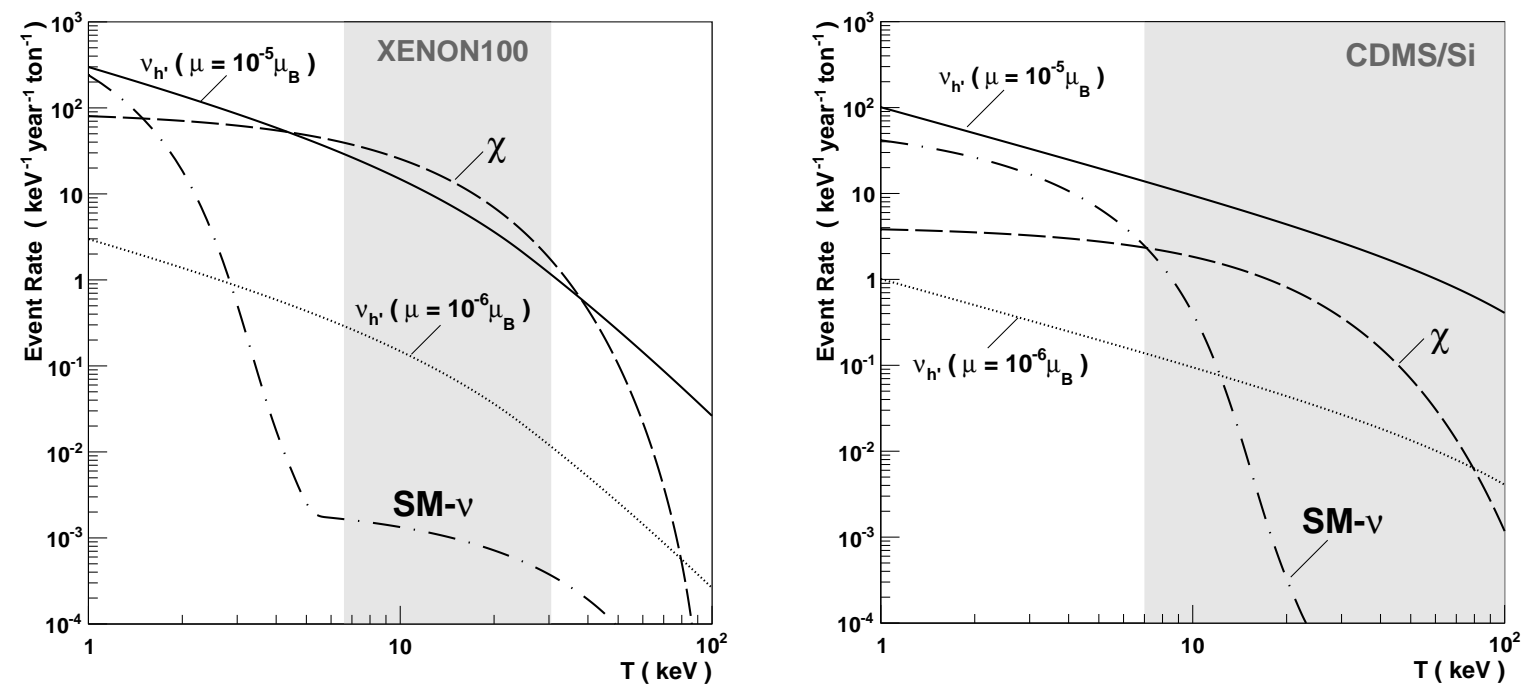

Figure 4: Recoil distribution of $\nu_{h^{\prime}}$ events for $\mu=10^{-6} \mu_{B}$ (dotted) and $\mu=10^{-5} \mu_{B}$ (solid) at XENON100 (left) and CDMS/Si (right). Also shown are the number of events from standard neutrino interactions (dot-dashed) as well as the current XENON100 limit 12] from a $55 \mathrm{GeV}$ WIMP with a cross section $\sigma_{\chi N}=2 \times 10^{-45} \mathrm{~cm}^{2}$ (dashed). The shaded regions show the acceptance regions of the experiments: XENON100 has $T \in[6,30] \mathrm{keV}$; CDMS/Si has $T \in[7,100] \mathrm{keV}$.

and CoGeNT.

To realize the scenario for DAMA/LIBRA, CoGeNT, CDMS/Si, and XENON100 that we have described in the previous section, we need $\mu_{h^{\prime}} \sim 10^{-2} \mu_{B}$ and also a much steeper flux, i.e., a mechanism that reduces the dipole energy on its way from the atmosphere to the detector and yields a spectral index $\gamma \approx 10$ deduced in Eq. (11). Remarkably, both effects may be correlated: a much larger value of $\mu_{h^{\prime}}$ could make the neutrino interact several times in the rock shielding the detector and, at the same time, would allow for the strong signal observed at DAMA/LIBRA and CoGeNT. More specifically, we estimate the energy loss through ionization of a dipole relative to that of a muon as

$$
-\left.\frac{d E}{d x}\right|_{N} \approx-\left.\frac{\mu^{2} m_{e}^{2}}{\pi \alpha}(\beta \gamma)^{2} \frac{d E}{d x}\right|_{\mu}
$$

with $\beta \gamma=p / m$ and $m_{e}$ the mass of the electron. For $\mu_{h^{\prime}}=10^{-6} \mu_{B}$ the stopping power of $1 \mathrm{~km}$ of rock would be negligible, while for values around $10^{-2} \mu_{B}$ the dipole would lose a significant fraction of energy. This latter value of $\mu_{h^{\prime}}$ would also be compatible with the DAMA/LIBRA and CoGeNT signals for a total dipole flux smaller than the atmospheric 

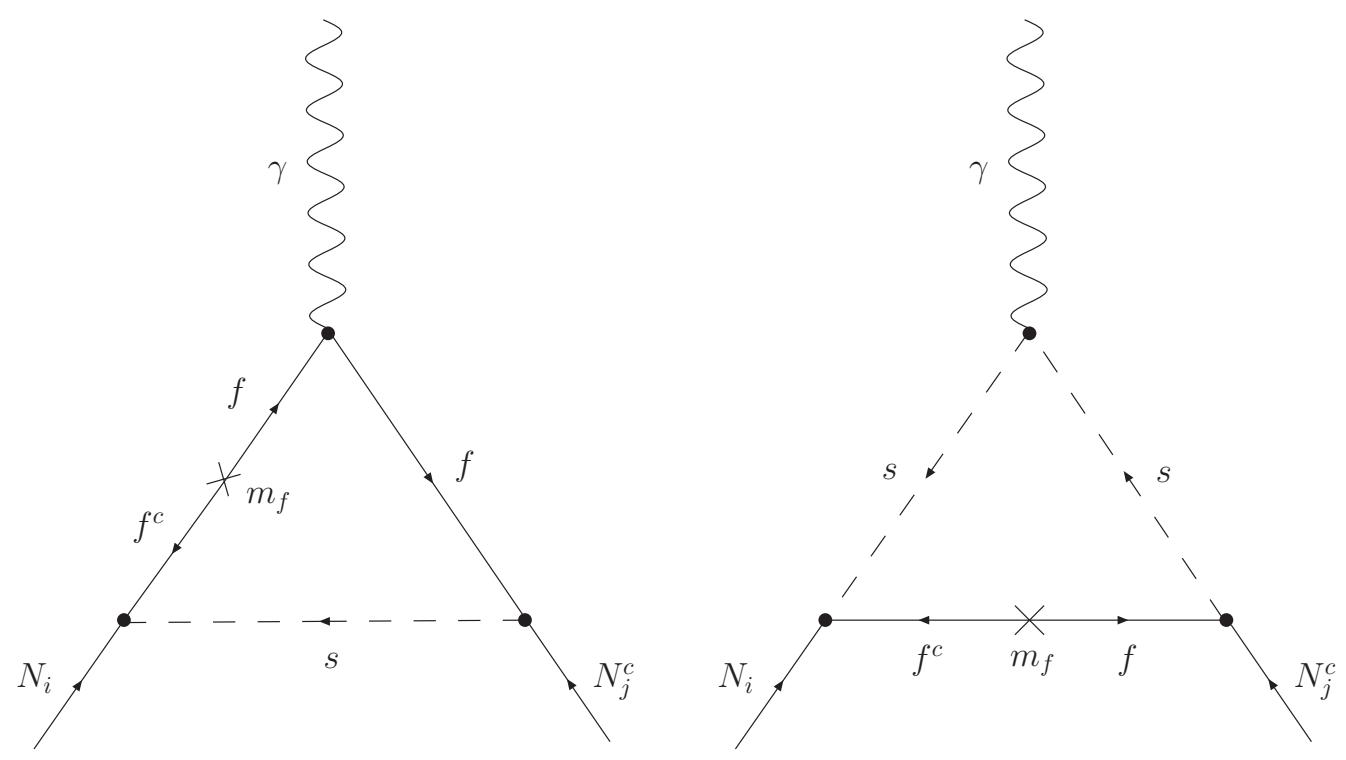

Figure 5: Some one-loop diagrams generating a magnetic dipole moment for the $\nu_{h^{\prime}}$ (composed of the two-component spinors $N$ and $N^{c}$ ) from the millicharged fermions $f$ and $f^{c}$ and the scalar $s$. The charges are $Q_{f}=-Q_{f^{c}}=Q_{s}=\epsilon e$ which permits the Yukawa couplings $f^{c} s N$ and $f s^{\dagger} N^{c}$.

neutrino flux (again, the number of events is proportional to $\Phi_{h^{\prime}} \times \mu_{h^{\prime}}^{2}$ ). Although a complete calculation of the $\nu_{h^{\prime}}$ flux at the detector site is beyond the scope of this work, we find interesting that both conditions are simultaneously satisfied for that value of $\mu_{h^{\prime}}$.

While a dipole moment of $10^{-2} \mu_{B}$ can explain the dark matter experiments, it also opens up the possibility of events at neutrino experiments measuring electron recoils. Employing Eq. (1) with $M=m_{e}$ yields that the maximum energy transfered to an electron is $T_{\max }=0.6$ $\mathrm{MeV}$ for an $m=10 \mathrm{MeV}, E=13 \mathrm{MeV}$ dipole. Such small electron recoils mean that $\nu_{h^{\prime}}$ would not be visible at MiniBooNE (where the anomaly is explained by the decays $\nu_{h} \rightarrow \nu_{h^{\prime}} \gamma$ of the heavier neutrino $\nu_{h}$ ), since the resulting electron energies would be well below the energy cuts. The atmospheric flux of $\nu_{h^{\prime}}$ would also be invisible in solar neutrino experiments like Super Kamiokande for the same reason: the energy threshold there is around $4 \mathrm{MeV}$. The remaining possibility of detection is then sub-MeV solar neutrino experiments like Borexino. We estimate that the atmospheric $\nu_{h^{\prime}}$ flux is around $10^{-10}$ that of the solar neutrino flux and that the ionization cross section of $\nu_{h^{\prime}}$ is about $10^{7}$ times larger than the quasielastic $\sigma\left(\nu_{e} A \rightarrow e A\right)$; therefore, the number of $\nu_{h^{\prime}}$ events at Borexino should be negligible.

Of course, we are still facing the fact that the required magnetic moments are much larger than the ones expected in natural completions of the model. We think, however, that such 
values are possible if, for example, our sterile neutrino $\nu_{h^{\prime}}$ is coupled to exotic particles of low mass (10-100 MeV) having electric millicharge. These particles should annihilate before primordial nucleosynthesis [35], and the massless paraphoton that they imply could give an interesting contribution to the dark radiation suggested by some experiments [36 39]. For example, one Dirac fermion $\left\{f, f^{c}\right\}$ plus one scalar $s$ both millicharged and with sizeable Yukawa couplings to $\left\{N_{h^{\prime}}, N_{h^{\prime}}^{c}\right\}$ could induce the large magnetic moment that is required through the diagrams in Figure 5. We estimate that these diagrams produce

$$
\mu \sim \frac{1}{16 \pi^{2}} \frac{y^{2} \epsilon}{m_{f}} \sim 10^{-2} \mu_{B}\left(\frac{\epsilon}{10^{-3}}\right)\left(\frac{10 \mathrm{MeV}}{m_{f}}\right)
$$

which is clearly around the desired magnetic moment.

\section{Summary and discussion}

We have shown how an atmospheric dipole may reconcile the seemingly inconsistent data of direct-search experiments, and we have provided a concrete model as evidence of its viability. We hope that the additional data of current and forthcoming experiments (ANAIS [40], COUPP [41], LUX [42], DEAP [43], etc.) will settle whether the current signals are due to DM, to new physics of atmospheric or solar [14, 15] origin, or to some unidentified background.

\section{Acknowledgments}

This work has been partially supported by MICINN of Spain (FPA2010-16802, FPA201017915, FPA2012-39489.C04.04 and Consolider-Ingenio Multidark CSD2009-00064 and CPAN CSD2007-00042), and by Junta de Andalucía (FQM 101,330,3048).

\section{References}

[1] DAMA Collaboration, R. Bernabei et al., Eur.Phys.J. C56, 333 (2008), arXiv:0804.2741.

[2] DAMA Collaboration, LIBRA Collaboration, R. Bernabei et al., Eur.Phys.J. C67, 39 (2010), arXiv:1002.1028. 
[3] CoGeNT collaboration, C. Aalseth et al., Phys.Rev.Lett. 106, 131301 (2011), arXiv:1002.4703.

[4] G. Angloher et al., Eur.Phys.J. C72, 1971 (2012), arXiv:1109.0702.

[5] CDMS Collaboration, R. Agnese et al., Phys.Rev.Lett. (2013), arXiv:1304.4279.

[6] LVD collaboration, M. Selvi, Analysis of the seasonal modulation of the cosmic muon flux in the lvd detector during 2001-2008, in Proceedings of the 31st International Cosmic Ray Conference, Eódź (Poland), July 7-15, 2009: Invited, Rapporteur and Highlight Papers, edited by J. Szabelski and M. Giller, University of Łódź. Department of High Energy Astrophysics, 2010.

[7] S. Chang, J. Pradler, and I. Yavin, Phys.Rev. D85, 063505 (2012), arXiv:1111.4222.

[8] D. Nygren, (2011), arXiv:1102.0815.

[9] K. Blum, (2011), arXiv:1110.0857.

[10] J. P. Ralston, (2010), arXiv:1006.5255.

[11] P. Sánchez-Lucas, On the influence of neutrino interactions in direct light wimps searches, masters thesis, University of Granada, 2012, (in Spanish).

[12] XENON100 Collaboration, E. Aprile et al., Phys.Rev.Lett. 109, 181301 (2012), arXiv:1207.5988.

[13] T. Araki et al., Nature 436, 499 (2005).

[14] R. Harnik, J. Kopp, and P. A. Machado, JCAP 1207, 026 (2012), arXiv:1202.6073.

[15] M. Pospelov and J. Pradler, Phys.Rev. D85, 113016 (2012), arXiv:1203.0545.

[16] S. Gninenko and N. Krasnikov, Phys.Lett. B450, 165 (1999), arXiv:hep-ph/9808370.

[17] CDMS-II Collaboration, Z. Ahmed et al., Science 327, 1619 (2010), arXiv:0912.3592.

[18] J. Conrad, Neutrino experiments, in Colliders And Neutrinos, edited by S. Dawson and R. N. Mohapatra, chap. 5, pp. 177-254, World Scientific, 2007, arXiv:0708.2446.

[19] LSND Collaboration, C. Athanassopoulos et al., Phys.Rev.Lett. 77, 3082 (1996), arXiv:nucl-ex/9605003.

[20] LSND Collaboration, C. Athanassopoulos et al., Phys.Rev. C54, 2685 (1996), arXiv:nucl-ex/9605001. 
[21] LSND Collaboration, C. Athanassopoulos et al., Phys.Rev.Lett. 81, 1774 (1998), arXiv:nucl-ex/9709006.

[22] LSND Collaboration, A. Aguilar-Arevalo et al., Phys.Rev. D64, 112007 (2001), arXiv:hep-ex/0104049.

[23] MiniBooNE Collaboration, A. Aguilar-Arevalo et al., Phys.Rev.Lett. 98, 231801 (2007), arXiv:0704.1500.

[24] MiniBooNE Collaboration, A. Aguilar-Arevalo et al., Phys.Rev.Lett. 102, 101802 (2009), arXiv:0812.2243.

[25] MiniBooNE Collaboration, A. Aguilar-Arevalo et al., Phys.Rev.Lett. 103, 111801 (2009), arXiv:0904.1958.

[26] MiniBooNE Collaboration, A. Aguilar-Arevalo et al., Phys.Rev.Lett. 105, 181801 (2010), arXiv:1007.1150.

[27] K. Abazajian et al., (2012), arXiv:1204.5379.

[28] S. N. Gninenko, Phys.Rev. D83, 015015 (2011), arXiv:1009.5536.

[29] M. Masip, P. Masjuan, and D. Meloni, JHEP 1301, 106 (2013), arXiv:1210.1519.

[30] KARMEN Collaboration, B. Armbruster et al., Phys.Rev. D65, 112001 (2002), arXiv:hep-ex/0203021.

[31] T2K Collaboration, K. Abe et al., Phys.Rev.Lett. 107, 041801 (2011), arXiv:1106.2822.

[32] M. Masip and P. Masjuan, Phys.Rev. D83, 091301 (2011), arXiv:1103.0689.

[33] R. Mohapatra and P. Pal, World Sci.Lect.Notes Phys. 41, 1 (1991).

[34] M. Honda, T. Kajita, K. Kasahara, S. Midorikawa, and T. Sanuki, Phys.Rev. D75, 043006 (2007), arXiv:astro-ph/0611418.

[35] S. Davidson and M. E. Peskin, Phys.Rev. D49, 2114 (1994), arXiv:hep-ph/9310288.

[36] Z. Hou et al., (2012), arXiv:1212.6267.

[37] WMAP Collaboration, G. Hinshaw et al., (2012), arXiv:1212.5226.

[38] Planck Collaboration, P. Ade et al., (2013), arXiv:1303.5076.

[39] J. L. Sievers et al., (2013), arXiv:1301.0824. 
[40] J. Amare et al., J.Phys.Conf.Ser. 375, 012026 (2012).

[41] COUPP Collaboration, E. Behnke et al., Nucl.Phys.Proc.Suppl. 221, 379 (2011).

[42] LUX Collaboration, S. Fiorucci, (2013), arXiv:1301.6942.

[43] DEAP Collaboration, M. Boulay, J.Phys.Conf.Ser. 375, 012027 (2012), arXiv:1203.0604. 